\title{
Recent Developments in Scanning Tunneling Spectroscopy of Semiconductor Surfaces
}

\author{
R. M. Feenstra, V. Ramachandran, and Huajie Chen \\ Department of Physics, Carnegie Mellon University, Pittsburgh, Pennsylvania \\ 15213 USA
}

\begin{abstract}
Several recent developments in scanning tunneling spectroscopy (STS) of semiconductor surfaces are reviewed. First, the normalization of spectra is discussed, which for the $\operatorname{Si}(111) 2 \times 1$ surface is found to produce a small shift in the apparent position of band edges. With this correction, the surface band gap measured by STS is found to be in good agreement with that obtained by other experimental and theoretical techniques. Second, it is shown for the $\operatorname{SiC}(0001) \sqrt{3} \times \sqrt{3}$ surface that the tunneling spectra show a remarkable evolution with decreasing current, and at pA levels they reveal a Mott-Hubbard gap for the surface states, in agreement with that seen by other methods. Finally, a detailed discussion is presented on the absence of electronic effects for the tunnel current into empty states of III-V (110) cleaved surfaces. From this result it is demonstrated that one can use observed strain induced displacements of such surfaces to yield information on the chemical composition of the underlying material.
\end{abstract}

\section{Introduction}

Since the early days of the scanning tunneling microscopy (STM), spectroscopy of semiconductor surfaces has played an important role both in furthering our understanding of the the STM technique as well as in yielding new information about the surfaces themselves. For example, early voltage-dependent results on $\mathrm{Si}(111) 2 \times 1$ and $\mathrm{Si}(111) 7 \times 7$ surfaces enabled a determination of their structures and also illustrated the exquisite sensitivity of the STM to the surface states [1-4]. Complete conductance spectra as a function of tip-sample bias voltage clearly revealed the surface state bands. This early development of the scanning tunneling spectroscopy (STS) field has been reviewed [5].

Slightly after the development of STS for semiconductor surface states, further studies were performed on cleaved GaAs(110) surface for which surface states do not play a large role in the results [6]. The difference in these two situations, with or without surface states, is quite dramatic. For $\mathrm{Si}(111) 7 \times 7$ the surface state band is essentially metallic (i.e. no band gap), and the conductance $d I / d V$ varies by less than three orders of magnitude over the voltage range -3 to $+3 \mathrm{~V}$. In contrast, for GaAs(110) a large band gap opens up in the spectrum and one requires 5-6 orders of magnitude in dynamic range to accurately measure the spectrum. Special techniques have been developed to achieve this high dynamic range [7]. For all of the direct gap III-V semiconductors, STS of the (110) surface can be used to reveal the bulk band gap as well as some other bulk critical point of the band structure [7]. Surface states make a small (but still noticeable) contribution of the spectra. 
In this paper we review several recent STS studies of semiconductor surfaces performed by our research group [8-10]. We first discuss two systems whose spectra are dominated by surface state effects, $\operatorname{Si}(111) 2 \times 1$ and $\operatorname{SiC}(0001) \sqrt{3} \times \sqrt{3}$. In the first case we show that a precise value of the surface band gap cannot be obtained by simply locating peaks in the normalized conductance, (dI/ $d V) /(I / V)$, but rather, a correction is required to those peak positions. With this correction, good agreement is found between the surface gap obtained from STS and those obtained from both a combination of photoemission (PES) [11] and inverse photoemission [12,13] and from recent many-body computations of the electronic structure [14]. In the second case we also discuss the surface state band gap, demonstrating that with exceptionally low current values one can observe a Mott-Hubbard gap in this system, with the size of the gap again agreeing with photoemission [15] and inverse photoemission [16] results as well as theoretical estimates [17]. We then discuss spectroscopic results obtained from III-V (110) surfaces, in which we argue that the electronic contribution to the tunnel current, for empty states imaged $2-3 \mathrm{eV}$ above the conduction band minimum, shows very little variation between the different III-V materials. Therefore, the constant-current STM images can be used as a relatively direct measure of actual topographic surface height, thereby enabling a direct determination of strain in semiconductor heterostructures based on the amount by which the surface is displaced due to elastic strain relaxation.

\section{Surface State Gap of $\mathrm{Si}(111) 2 \times 1$}

The $2 \times 1$ reconstruction of the $\mathrm{Si}(111)$ surface, formed by cleaving in ultra-high-vacuum conditions, has been a prototypical system in both experimental and theoretical studies for many years. It is now well accepted that the $\pi$-bonded chain model correctly describes the atomic arrangement of the surface [18], although a full understanding of the electronic structure, including excitonic effects, is still evolving [19,20]. This surface has provided an important test case for many-body computations of the surface-state band gap [20]. Experimentally, the surface-state gap of $\mathrm{Si}(111) 2 \times 1$ was observed in early work using optical absorption spectroscopy,[21] and was found to be about $0.45 \mathrm{eV}$ wide. Scanning tunneling spectroscopy (STS) measurements yielded a value for the surface gap of $0.50 \pm 0.05 \mathrm{eV}[2,4,22,24]$. A combination of photoemission and inverse photoemission spectroscopy (PES/IPES) produced a value of 0.75 for this gap $[11,12]$, which was later refined to about $0.60 \mathrm{eV}$ based on computations involving the finite resolution of the IPES spectrometer [13]. Because of the above mentioned importance of this surface in providing a test case for theoretical work, it is important to obtain the most accurate possible values for the surface band gap from the various experiments. For this reason, we have considered a particular correction to the tunneling spectroscopy result, which produces an increase by nearly $0.1 \mathrm{eV}$ of the value directly observed in the experiments.

In STS studies, one measures the tunnel current $I$ and differential conductance $d I / d V$ as a function of the sample-tip voltage $V$. It is then conventional to compute a normalized conductance, $(d I / d V) /(I / V)$, and to compare this quantity to an expected surface density of states (DOS) [4,22]. This normalized conductance provides a convenient means of characterizing the observed spectrum, yielding e.g. sharp, peaked features near the onsets of surface bands which provide a more well-defined indicator of the onset position than the relatively gradual turn-on of the current or differential conductance. Nevertheless, some deviations between the normalized conductance and the surface DOS can occur. To estimate these effects for the $\operatorname{Si}(111) 2 \times 1$ surface we have performed an explicit computation of the normalized conductance, starting from a model for the density of states of the surface bands [19]. From the tunnel current obtained by the usual formula [9], we nu- 
merically compute the differential conductance $d I / d V$, and the normalized conductance $(d I / d V) /(I /$ $V)$.

Figure 1 shows the tunnel current and normalized conductance resulting from the assumed surface DOS. The onset of the surface band is taken to be at $0.3 \mathrm{eV}$. We use a tip-sample separation of $6 \AA$, and average work-function of $4 \mathrm{eV}$. By examination of the computed curve for $(d I / d V) /(I /$ $V)$ it is clear that the maximum in this quantity occurs at a voltage significantly less than $0.3 \mathrm{~V}$; the peak position is shifted by $-0.047 \mathrm{~V}$ below the band edge. This value is not strongly dependent on the parameters used in the computations: varying the tip-sample separation or the work-function by $30 \%$ produces a change in the shift of less than $0.001 \mathrm{~V}$, and similarly choosing a different model for the barrier (i.e. including the image potential) produces very little effect. If we choose a different form for the surface DOS, e.g. a step function with no dependence on $k_{\|}$, the shifts are nearly the same of for the $\pi$-bonded chain DOS. The computed shifts vary approximately linearly with the assumed band edge position of the surface bands. Results for negative bias voltages (energies) are very similar to those for positive bias.

For the case of the $\operatorname{Si}(111) 2 \times 1$, with band onsets at -0.3 and $+0.2 \mathrm{~V}$, we find a total correction to the band gap value of about $0.080 \pm 0.02 \mathrm{eV}$ [8]. Combining this result with our best value of the band gap from peaks $(d I / d V) /(I / V)$ based on a reanalysis of the data of Ref. [4], $0.51 \pm 0.03$ $\mathrm{eV}$ [23], yields a corrected gap value of $0.59 \pm 0.04 \mathrm{eV}$. This value should be compared with the minimum band gap on the surface, which appears to be indirect for the case of Si(111)2×1 [20,24]. Our result is in good agreement with the theoretical indirect gap of $0.58 \mathrm{eV}$ [20], and also compares favorably with the PES/IPES results of $0.60 \mathrm{eV}$ for the direct gap [11-13] (about $0.04 \mathrm{eV}$ high then the indirect gap according to theory [20]). Our results thus support the current view of excitonic effects accounting for the $\approx 0.15 \mathrm{eV}$ difference between these energies and the optical absorption onset of $0.45 \mathrm{eV}[20,21]$.

\section{Mott-Hubbard Gap of $\operatorname{SiC}(0001) \sqrt{ } 3 \times \sqrt{ } 3$ Surface}

Silicon carbide is a wide bandgap semiconductor with properties that make it useful in high temperature electronics and other areas. Several workers have studied the atomic structure of the surface using the scanning tunneling microscope (STM) $[25,26]$. A set of reconstructions of the Si terminated (0001) surface has been discovered, one of which, the $\sqrt{3} \times \sqrt{3}$, has evoked much experimental [15,16,27] and theoretical [17,28-30] interest. Theoretically, the lowest energy model for this reconstruction consists of $\mathrm{Si}$ adatoms at $\mathrm{T}_{4}$ positions on a Si terminated bulk crystal. Here, three of the Si adatom bonding orbitals are back-bonded to Si atoms. The fourth bonding orbital extends into vacuum with only one electron in it. Local density functional calculations for this structural model $[28,29]$ predict a half-filled, and hence metallic band arising from the dangling bond. More refined computations employed a two-dimensional Hubbard model and indicated that the energy levels of this surface consist of a filled and an empty band, separated by a Hubbard gap of $U=1.6 \mathrm{eV}$, thus producing a semiconducting density of states (DOS). Such a surface would have at each site a single localized electron, forming a 2-D system of spins which can take two values on a triangular lattice. This problem is of great theoretical interest because such systems can be frustrated and form 2-D spin glasses. This structure has been questioned in the literature, and several alternatives have been proposed $[25,27,31,32]$.

Photoemission spectroscopy (PES) experiments on n-type 6H-SiC (0001) wafers which 
show a $\sqrt{3} \times \sqrt{3}$ low energy electron diffraction (LEED) pattern reveal a fully-filled band $1.2 \mathrm{eV}$ below the Fermi level [15]. Inverse photoemission spectroscopy (IPES) [16] on the same surface shows an empty surface state $1.1 \mathrm{eV}$ above the Fermi level. Both these results are in moderate agreement with recent theoretical predictions of a Mott-Hubbard ground state for this surface [17] as mentioned above. Scanning tunneling spectroscopy (STS) has a great advantage over PES and IPES in that a single tunneling spectrum can show surface states both above and below the Fermi level simultaneously, at the same spatial location. In this work we have measured tunneling spectra for the $\sqrt{3} \times \sqrt{3}$ structure for both $\mathrm{p}$-type and n-type $6 \mathrm{H}$-SiC. We find that at typical tip-sample separations given by a tunneling current setpoint of $100 \mathrm{pA}$, the tunneling spectra show no resemblance to the PES and IPES results. But as the tip is retracted from the surface by reducing the tunnel current setpoint, features of the density of states seen in these other experiments begin to appear and several other strong features seen only in the high current tunneling spectra get suppressed. We have measured tunneling spectra over four orders of magnitude of current setpoint values going down to as low as $0.5 \mathrm{pA}$ in some cases, and traced the evolution of the density of states through them. We observe a remarkable evolution of the spectra with current. At high currents we see the appearance of doping-dependent features in the spectra which we attribute to tip-induced band bending. Using exceptionally low currents, we observe a filled state $1 \mathrm{eV}$ below the Fermi level and an empty state $1 \mathrm{eV}$ above, in agreement with both prior experiment and theory. Also, importantly, STM images of the surface taken when tunneling into the empty state or out of the filled state show that both the empty and filled states are localized at the same spatial point, further supporting a Mott-Hubbard type band structure for the surface.

$\mathrm{SiC}(0001)$ surfaces for our experiments are prepared as previously described [9]. Figure 2(a) shows a spectrum obtained on n-type $6 \mathrm{H}-\mathrm{SiC}$, acquired with a tunnel current setpoint of 100 $\mathrm{pA}$, a value which is typical of STM experiments (The current setpoint corresponds to the constant current used prior to acquiring the spectrum, which generally is close to the value of tunnel current at the positive and/or negative end of the spectrum. Measurable values of the current in the spectra presented here range over 2-3 orders of magnitude below the setpoint). In Fig. 2(b) we show the main features of the PES and IPES results on the $\sqrt{3} \times \sqrt{3}$ surface. It is apparent that the agreement between the STS and PES/IPES results is poor. The tunneling spectrum shows a small bandgap of about $0.6 \mathrm{eV}$, compared to $2.3 \mathrm{eV}$ in the combined PES/IPES spectra. The tunneling spectrum is almost featureless in the range where the surface states are expected, and at more negative voltages we see a large feature D1 which has no counterpart in the PES spectrum. Because of the increased barrier to tunneling from states below the Fermi level, tunneling spectra features at negative $V$ should be suppressed, but remarkably this feature seems to be very strong. Nearly identical spectra at similar setpoint values have been obtained by other workers [33].

Figures 3(a) and (b) show a series of spectra acquired with varying current setpoints for (a) n-type material and (b) p-type material. As the setpoint is reduced and the STM tip withdrawn, several dramatic effects are noticed. For n-type material, the large feature D1 at negative voltages diminishes. Two new states appear above the Fermi level, at $E-E_{F}=+0.9 \mathrm{eV}$ and $+1.9 \mathrm{eV}$, and a state also appears below the Fermi level at $E-E_{F}=-1.1 \mathrm{eV}$. The states at about $1 \mathrm{eV}$ above and below the Fermi level we identify with those seen in PES and IPES. The origin of the state observed at $1.9 \mathrm{eV}$ above $E_{F}$ is not clear at present, although it may be associated with one of the many surface resonances which occur on this surface [29]. For p-type material, at large currents we again see a mostly featureless spectrum with a strong feature $\mathrm{D}_{2}$ at large positive voltages. As the tip is retract- 
ed, this distinctive feature vanishes and the two states at positive voltages and one at about $-1 \mathrm{~V}$ appear. The position of these states is shifted by $0.2 \mathrm{eV}$ relative to the n-type results due to a shift in the surface Fermi level position, which is clearly evident by comparing the 1 pA spectra in Figs. 3(a) and (b).

Clearly, at large tunnel currents the spectra obtained are not intrinsic properties of the surface itself but arise from effects of the STM probe tip on the surface. These observed effects scale with tunnel current, and we attribute them to a type of "spreading resistance" in the transport of carriers to (or from) the localized surface states [34]. Such transport may occur through subsurface bulk states or through surface states, but in either case it appears from the data that at the relatively large current values, limited mobility of carriers results in the formation of a voltage drop on the surface region below the tip apex [9].

Explanation of the high voltage, doping dependent features $\mathrm{D}_{1}$ and $\mathrm{D}_{2}$ requires additional considerations. First we recall the occurrence of the "dopant induced" components of tunneling in semiconductors with no surface states, in which, at negative sample bias for n-type material, electrons in the conduction band can tunnel out of the material, and similarly for holes in the valence band seen for positive voltage in p-type material [6]. Such features are not expected (and have never been reported) when a significant number of surface states are present, since the states generally pin the surface Fermi level position at a midgap position thereby pinching off these dopant-induced components [6]. However, with the spreading resistance type of effect described above, the surface Fermi level position will be different than that in the bulk, so that the bulk bands can flatten out and produce this dopant induced source of current. For the case of $\operatorname{SiC}(0001) \sqrt{3} \times \sqrt{3}$, the surface Fermilevel (at zero tunnel current) is pinned at a location $0.6 \pm 0.2 \mathrm{eV}$ below the conduction band edge for n-type material [15]. We then expect, in the limit of large tunnel current, that a dopant-induced component will be seen for negative voltage with magnitude larger than $0.6 \pm 0.2 \mathrm{~V}$. This result is close to that seen in the $1 \mathrm{nA}$ spectra of Fig. 3(a) in which the onset of the $\mathrm{D}_{1}$ component is at about $-1 \mathrm{~V}$. Similarly, for p-type material with a pinning position of $0.8 \pm 0.2 \mathrm{eV}$ below the conduction band edge, and using the bandgap of $2.9 \mathrm{eV}$, we expect a dopant-induced component at positive voltage greater than $2.1 \pm 0.2 \mathrm{~V}$. This result again agrees with the onset of $\mathrm{D}_{2}$ at $+2.2 \mathrm{~V}$ in Fig. 3(b).

Based on our spectra, we conclude that, in agreement with PES and IPES experiments, we see surface states at about the same energies as predicted by theory. The strong appearance of spreading resistance effects also shows that carrier transport is very limited and so these surface bands are indeed narrow. However, it is important to realize that an electronic band structure alone cannot unambiguously determine the actual surface structure (many semiconductor surfaces show a gap in the surface DOS). Along with the acquisition of spectra, voltage dependent imaging of the surface is necessary to determine the spatial location of these states. In Fig. 4 we show topographic views of the $6 \mathrm{H}-\mathrm{SiC}(0001) \sqrt{3} \times \sqrt{3}$ reconstruction on n-type material. Figure 4(a) was acquired at a sample voltage of $-1.2 \mathrm{~V}$ and a current setpoint of $100 \mathrm{pA}$. Imaging at positive voltages at this setpoint was very unstable, as expected from the absence of features in the spectra above the Fermi level. Figures 4(b) and (c) were acquired simultaneously (alternating line scans) at $-1 \mathrm{~V}$ and $+1 \mathrm{~V}$ respectively with a setpoint of $10 \mathrm{pA}$. As seen there, the topographic maxima at both polarities are at the same spatial location, thus demonstrating that the states corresponding to both the band $1 \mathrm{~V}$ above the Fermi level and the band $1 \mathrm{~V}$ below the Fermi level are located at the same position on the surface. Similar results are obtained for sample voltages with magnitudes in the range 0.8-2.0 
V. This combination of tunneling spectra and surface topography is clearly consistent with what is expected for $\mathrm{a} \mathrm{T}_{4} \mathrm{Si}$ adatom model. Furthermore, it is inconsistent with other models of the surface which involve vacancies [27], trimers [25,29] or more complicated entities since there, we would expect either metallic bands or the empty and filled states at different spatial locations or both. Our results are unable to distinguish between $\mathrm{Si}$ and $\mathrm{C}$ adatoms, $\mathrm{T}_{4}$ and $\mathrm{H}_{3}$ adatoms, or discern rearrangements of the bulk layers beneath the adatoms, but nevertheless are strongly in favor of a MottHubbard model since the empty and filled states are spatially coincident.

\section{Cross-sectional STM of III-V Semiconductors}

As discussed in Section I, the (110) surfaces of the III-V semiconductors such as GaAs are not reconstructed, so that STM/STS studies of such surfaces yield information which is not particularly surface specific. Thus, one can usefully study heterostructures of such materials, in which the structures are cleaved on the (110) or (110) plane and viewed in cross-section thereby revealing the interfaces between layers as well as detailed properties of the layers themselves. Such studies are generally referred to as cross-sectional STM (xSTM), and they have been performed by many groups to obtain atomic-scale information regarding the structure of III-V heterostructures [35-38].

An example of a recent XSTM study performed in our research group is given in Fig. 5, which shows an $\mathrm{In}_{0.53} \mathrm{Ga}_{0.47} \mathrm{As}$ quantum well embedded between InP barrier layers [39]. Figure 5(a) shows the as-grown heterostructure, and Fig. 5(b) shows the structure after ion implantation and subsequent annealing [39]. The ion implantation causes intermixing between the quantum well and the barrier layers. This intermixing, in turn, produces a blue shift in the optical transition energy of the quantum well, and thus the quantum well intermixing process can be used for tuning the emission wavelength of lasers and for other photonic integrated device applications [40]. The intermixing between quantum well and barrier layers is clearly evident in Fig. 5(b): we see some white (higher tip height) bands forming at the well/barrier interfaces, and the quantum well itself is darker (lower tip height) in Fig. 5(b) compared to Fig. 5(a). For the particular choice of implantation parameters used in this case [39] we have apparently produced a thin region near the well/ barrier interface with new chemical composition which gives the white contrast in the STM images, along with modifying the overall quantum well composition thereby producing its darker contrast.

Given STM data of the type shown in Fig. 5, it is desirable to obtain a quantitative evaluation of chemical composition as a function of position in the heterostructure. Such an evaluation is, of course, quite difficult in general since the contrast in STM images contains significant contributions from electronic effects in the tunnel current and such effects are not easily quantified. However, in a series of studies of strained heterostructures, we have recently observed a second significant contribution to STM images, arising from displacement of the (110) cleavage face due to strain in the underlying material [41-43]. In cases where electronic effects can be shown to be small, this mechanical or elastic contribution to the STM contrast can be relatively simply evaluated using a numerical solution (finite element method) of the elasticity equations, thus providing a means of determining strain and associated chemical composition in the heterostructure.

Figure 6 illustrates these electronic and mechanical contributions to the STM contrast for the case of a semiconductor superlattice with compressively strained barriers and tensilely strained quantum well layers. In this particular case, the respective contributions have opposite sign, al- 
though for compressive wells with tensile barriers the electronic and mechanical effects would have the same sign. Let us consider the magnitude of the mechanical effect. As noted in the caption of Fig. 6, the peak-to-peak corrugation amplitude is approximately equal to $2 \varepsilon L$ where $L$ is the width of barrier and well, and their strains (in-plane, diagonal component) are $\pm \varepsilon$. For example, with $5 \mathrm{~nm}$ thick well and barrier, having strains (in plane, diagonal components) of \pm 0.01 , the amplitude would be $1 \AA$. Offhand, this result would appear to be of the same order as typical electronic effects in the tunnel current between different materials, so it may seem difficult to distinguish between electronic and mechanical effects. However, a more detailed analysis reveals that the electronic effects are, in many cases, sufficiently small so that they can be neglected relative to the mechanical effects of the strain relaxation.

Let us consider the tunnel currents in the materials pictured in Fig. 7. The separation between conduction band minimum and tip Fermi level is larger for the material with smaller band gap, which would imply a greater number of states available for tunneling and hence a larger tunnel current in that case compared to the larger band gap material. However, this conclusion is valid only for relatively small bias voltages between tip and semiconductor. For larger voltages, typical$1 y \geq 2 \mathrm{~V}$, one must explicitly include consideration of the voltage-dependence of the transmission term for tunneling through the vacuum region, as pictured by the function $D(E, V)$ in Fig. 7. Assuming a simple trapezoidal barrier, this function can be written down in the standard manner [10]. It is well known that the energy and voltage dependence of $D(E, V)$ is such that the highest lying states are favored in the tunneling process [44]. For $V>0$ the maximum of $D(E, V)$ occurs at $E=e \cdot V$, and for $V<0$ the maximum is at $E=0$. The former case is illustrated in Fig. 7. The rate of decrease of $D(E, V)$ with decreasing $E$ depends on the work function and tip-sample separation. For typical values of $\bar{\vartheta}=4 \mathrm{eV}$ and $s=6 \AA, D(E, V)$ changes by a factor of 20 as $E$ varies over a $2 \mathrm{eV}$ interval. Thus, for tunneling voltages larger than about $2 \mathrm{~V}$, increasing the voltage does not lead to a significant difference in the width of the energy window for availability of states for tunneling, and so an increase in the tunnel current is not expected. However, the $e \cdot V / 2$ term in the barrier equation [10], arising from the decrease in the average barrier height with increasing voltage, will still produce an overall increase in the current with increasing voltage.

To evaluate the tunnel current, band structure computations are performed using empirical nonlocal pseudo-potentials. Spin-orbit coupling is not included. We focus on the materials GaAs, InP, InAs, and GaP which form the endpoints of the In $x \mathrm{Ga} 1-x \mathrm{As} y \mathrm{P} 1-y$ alloy system, since these alloys are most relevant to our prior xSTM measurements $[39,43]$. Results for the band structure and density of states of these materials are very similar to those given previously by Fischetti [45]. The tunnel current is computed by numerically evaluating the usual integral containing a sum over states of the perpendicular velocity of the electrons [10]. It is well known from early studies of tunneling that the effects of this velocity term are largely cancelled by a density of states term which appears when the integral over states is performed, so that the current shows very little dependence on the band structure (i.e. on the chemical composition) of the material [46].

Results for tunneling into conduction band (CB) states are shown in Fig. 8. As seen there, at low voltages the tunnel current does vary considerably between the various materials. However, at higher voltage $>3 \mathrm{~V}$, the current is practically the same amongst the different materials. We find less than a factor of 1.5 variation in the tunnel current, corresponding to a tip height variation of < $0.20 \AA$. We note that the zero of voltage in Fig. 8 is taken to be the CB minimum of InAs. In a 
typical experiment it would be at a different point, i.e. the lowest-lying conduction band minimum of the relevant alloy material, typically $0.5 \mathrm{~V}$ higher than that shown in Fig. 8. In that case, the voltage range for which the tunnel currents converge would be $>2.5 \mathrm{~V}$. Furthermore, we consider experiments in which the In $x \mathrm{Ga} 1-x \mathrm{As} y \mathrm{P} 1-y$ alloy compositions used are in a rather restricted range, which is quite far from $\mathrm{GaP}[39,43]$. In that case, the expected variation in tip height variation would be less, typically $\lesssim 0.07 \AA$, and the minimum voltage needed to achieve this convergence of the tunnel currents is also less, typically $\gtrsim 2.0 \mathrm{~V}$. This voltage range is well within the parameters of usual xSTM experiments on In $x \mathrm{Ga} 1-x \mathrm{As} y \mathrm{P} 1-y$ alloys, which typically employ sample voltages with magnitude in the range $2.0-2.5 \mathrm{~V}$. Values significantly less than this are generally avoided since they can lead to tip-sample contact, and values much larger than this are also dangerous since they may lead to material transfer between tip and sample.

We conclude that for large, positive voltages that the tunnel current expected from In $x \mathrm{Ga}$ $1-x \mathrm{As} y \mathrm{P} 1-y$ alloys of any composition will be nearly the same. Less than a factor of 2 variation is found between GaAs, InP, InAs, and GaP materials, corresponding to a tip height variation in the STM of $<0.3 \AA$. Typically in xSTM experiment one encounters alloy variations which span only a considerably restricted subset of the total range between GaAs, InP, InAs, and GaP. In that case, the expected variation in tip height due to electronic effects would be smaller, typically $\lesssim 0.1 \AA$. As discussed above, the relatively small size of this electronic contribution to the tunnel current then enables the possibility of quantitatively determining strain variations in the heterostructures from the observed strain induced displacement of the cleavage face.

\section{Summary}

In summary, we have considered several different cases of STS on semiconductor surfaces. For the $\operatorname{Si}(111) 2 \times 1$ and the $\operatorname{SiC}(0001) \sqrt{3} \times \sqrt{3}$ surfaces, surface electronic states dominate the spectrum. The normalized conductance, $(d I / d V) /(I / V)$ is used to obtain a measure of the surface state density, although for the very precise values needed for the $\mathrm{Si}(111) 2 \times 1$ surface some small corrections to this quantity were required. For III-V (110) surfaces it is argued that surface states make only a small contribution of the tunnel current, and furthermore, that the tunnel current into bulk states depends only weakly on the chemical composition of the semiconductor. In that case, it is demonstrated that constant-current STM images can provide a useful measure of strain induced displacement of the surface in semiconductor heterostructures.

\section{Acknowledgments}

We acknowledge useful discussions with S. G. Louie, P. Mårtensson, J. E. Northrup, and C. G. Van de Walle. We are grateful to Max Fischetti for supplying the band structure code used in the computations discussed in Section IV. This work was supported by the National Science Foundation under grant DMR-9985898.

[1] R. S. Becker, J. A. Golovchenko, D. R. Hamann and B. S. Swartzentruber, Phys. Rev. Lett. 55, 2032 (1985).

[2] R. M. Feenstra, W. A. Thompson, and A. P. Fein, Phys. Rev. Lett. 56, 608 (1986).

[3] R. J. Hamers, R. M. Tromp, and J. E. Demuth, Phys. Rev. Lett. 56, 1972 (1986).

[4] J. A. Stroscio, R. M. Feenstra, and A. P. Fein, Phys. Rev. Lett. 57, 2579 (1986).

[5] R. M. Feenstra, Surf. Sci. 299/300, 965 (1994). 
[6] R. M. Feenstra and J. A. Stroscio, J. Vac. Sci. Technol. B 5, 923 (1987).

[7] R. M. Feenstra, Phys. Rev. B 50, 4561 (1994).

[8] R. M. Feenstra, Phys. Rev. B 60, 4478 (1999).

[9] V. Ramachandran and R. M. Feenstra, Phys. Rev. Lett. 82,1000 (1999).

[10] R. M. Feenstra, Physica B 273-274, 796 (1999).

[11] R. I. G Uhrberg, G. V. Hansson, J. M. Nicholls, and S. A. Flodstrom, Phys. Rev. Lett. 48, 1032 (1982).

[12] P. Perfetti, J. M. Nicholls, and B. Reihl, Phys. Rev. B 36, 6160 (1987).

[13] A. Cricenti, S. Selci, K. O. Magnusson, and B. Reihl, Phys. Rev. B 41, 12908 (1990).

[14] M. Rohlfing and S. G. Louie, Phys. Rev. Lett. 83, 856 (1999).

[15] L. I. Johansson, F. Owman and P. Mårtensson, Surf. Sci. 360, L478 (1996).

[16] J.-M. Themlin, I. Forbeaux, V. Langlais, H. Belkhir and J.-M. Debever, Europhys. Lett. 39, 61 (1997).

[17] J. E. Northrup and J. Neugebauer, Phys. Rev. B 57, R4230 (1998).

[18] K. C. Pandey, Phys. Rev. Lett. 47, 1913 (1981); Phys. Rev. Lett. 51, 17 (1985).

[19] R. Del Sole and A. Selloni, Phys. Rev. B 30, 883 (1984), Eq. (13). Note that there is a typographical error in this equation, the cosine term should be squared.

[20] J. E. Northrup, M. S. Hybertsen, and S. G. Louie, Phys. Rev. Lett. 66, 500 (1991); Proceedings of the 20th International Conference on the Physics of Semiconductors, editors J. D. Joannopoulos and E. M. Anastassakis (World Scientific, Teaneck, 1990), p. 119.

[21] G. Chiarotti, S. Nannarone, R. Pastore, and P. Chiaradia, Phys. Rev. B 4, 3398 (1971).

[22] R. M. Feenstra, J. A. Stroscio, and A. P. Fein, Surf. Sci. 181, 295 (1987).

[23] Band edge locations in Ref. [4] are given as $-0.3 \mathrm{eV}$ and $0.2 \mathrm{eV}$. A careful reexamination of the data reveals more precise values as being $-0.31 \pm 0.02 \mathrm{eV}$ and $0.20 \pm 0.02 \mathrm{eV}$ respectively. The difference between these values is $0.51 \pm 0.03 \mathrm{eV}$, where the error estimate in the result is computed by combining the component errors in quadrature.

[24] R. M. Feenstra, Phys. Rev. B 44, 13791 (1991).

[25] F. Owman and P. Mårtensson, Surf. Sci. 330, L639 (1995).

[26] L. Li and I. S. T. Tsong, Surf. Sci. 351, 141 (1996).

[27] L. Li, T. Sakurai and I. S. T. Tsong, J. Appl. Phys. 80, 2524 (1996).

[28] J. E. Northrup and J. Neugebauer, Phys. Rev. B 52, R17001 (1995).

[29] M. Sabisch, P. Krüger and J. Pollmann, Phys. Rev. B 55, 10561 (1997).

[30] S. Scandolo, F. Ancilatto, G. L. Chiarotti, G. Santoro, S. Serra and E. Tosatti, Surf. Sci. 402404, 808 (1998).

[31] T. Tsukamoto, M. Hirai, M. Kusaka, M. Iwami, T. Ozawa, T. Nagamura, T. Nakata, Surf. Sci. 
371, 316 (1997).

[32] P. Badziag, Surf. Sci. 402-404, 822 (1998).

[33] F. Owman and P. Mårtensson, unpublished.

[34] F. Flores and N. Garcia, Phys. Rev. B 30, 2289 (1984).

[35] For review, R. M. Feenstra, Semicond. Sci. Technol. 9, 2157 (1994).

[36] For review, E. T. Yu, Materials Science \& Engineering R: Reports, R17, 147 (1996).

[37] O. Albrektsen, D. J. Arent, H. P. Meier, and H. W. M. Salemink, Appl. Phys. Lett. 57, 31 (1990).

[38] S. Gwo, K.-J. Chao, C. K. Shih, Appl. Phys. Lett., 64, 493 (1994).

[39] H. Chen, R.M. Feenstra, P.G. Piva, R.D. Goldberg, I.V. Mitchell, G.C.Aers, P.J. Poole and S. Charbonneau, Appl. Phys. Lett. 75, 79 (1999).

[40] S. Charbonneau, P. J. Poole, P. G. Piva, G. C. Aers, E. S. Koteles, M. Fallahi, J.-J. He, J. P. McCaffrey, M. Buchanan, M. Dion, R. D. Goldberg, and I. V. Mitchell, J. Appl. Phys. 78, 3697 (1995).

[41] T. Pinnington, A. Sanderson, T. Tiedje, T.P. Pearsall, E. Kasper, H. Presting, Thin Solid Films, 222, 259 (1992).

[42] H. Chen, R. M. Feenstra, R. S. Goldman, C. Silfvenius, and G. Landgren, Appl. Phys. Lett. 72, 1727 (1998).

[43] B. Grandidier, R. M. Feenstra, C. Silfvenius, and G. Landgren, J. Vac. Sci. Technol. A 17, 2251 (1999).

[44] R. M. Feenstra, J. A. Stroscio, and A. P. Fein, Surf. Sci. 181, 295 (1987).

[45] M. V. Fischetti, IEEE Trans. Electron Devices 38, 634 (1991).

[46] C. B. Duke, Tunneling in Solids (Academic, New York, 1969), Eq. (7.8b) and discussion following. 


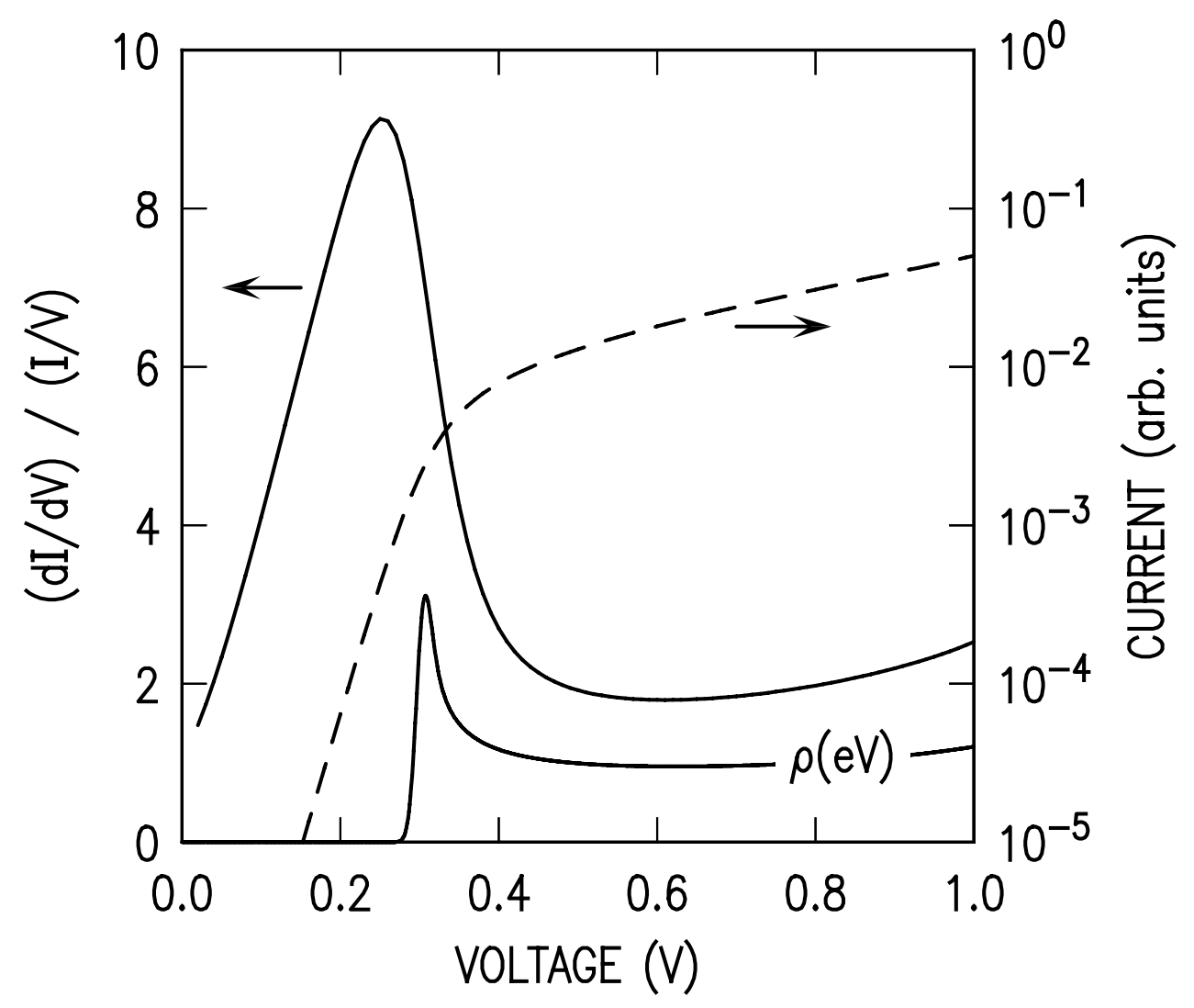

Figure 1 Computed tunnel current (dashed line) and normalized conductance $(d I / d V) /(I / V)$ (solid line), for the assumed surface DOS, $\rho(e V)$, for $\pi$-bonded chains as shown in the lower part of the figure. For plotting purposes, the surface DOS has been broadened using a Gaussian with FWHM of $0.02 \mathrm{eV}$. From Ref. [8]. 


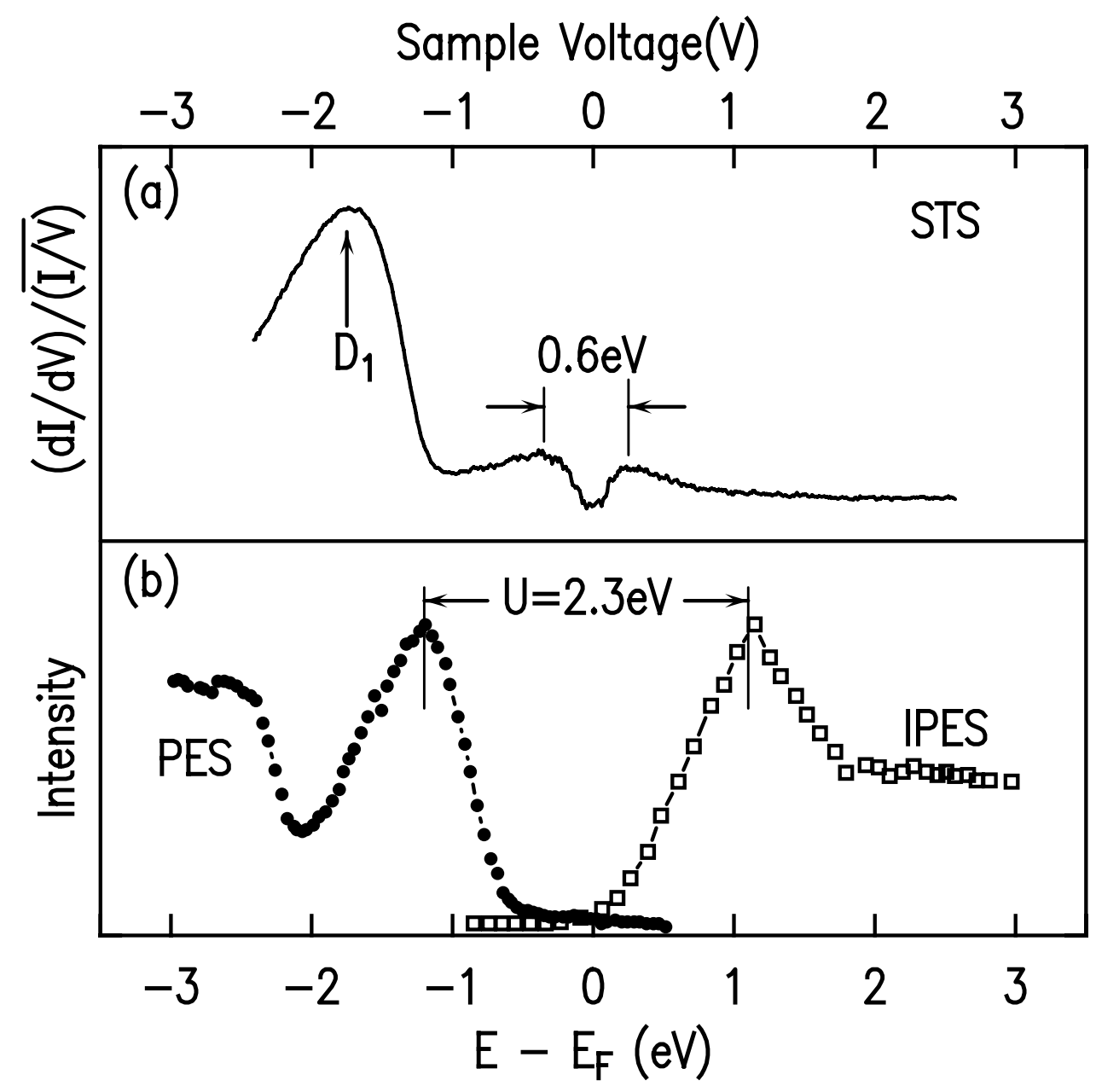

Figure 2 (a) Tunnel spectrum at a current setpoint of 100 pA (measurable values of the tunnel current in the spectrum range over 2-3 orders of magnitude below the setpoint). (b) Results of PES and IPES experiments, taken from from Refs. [15] and [16] respectively. The zero level for (dI/ $d V) /(\overline{I / \mathrm{V}})$ in (a) is given by the horizontal line separating panels (a) and (b). From Ref. [9]. 


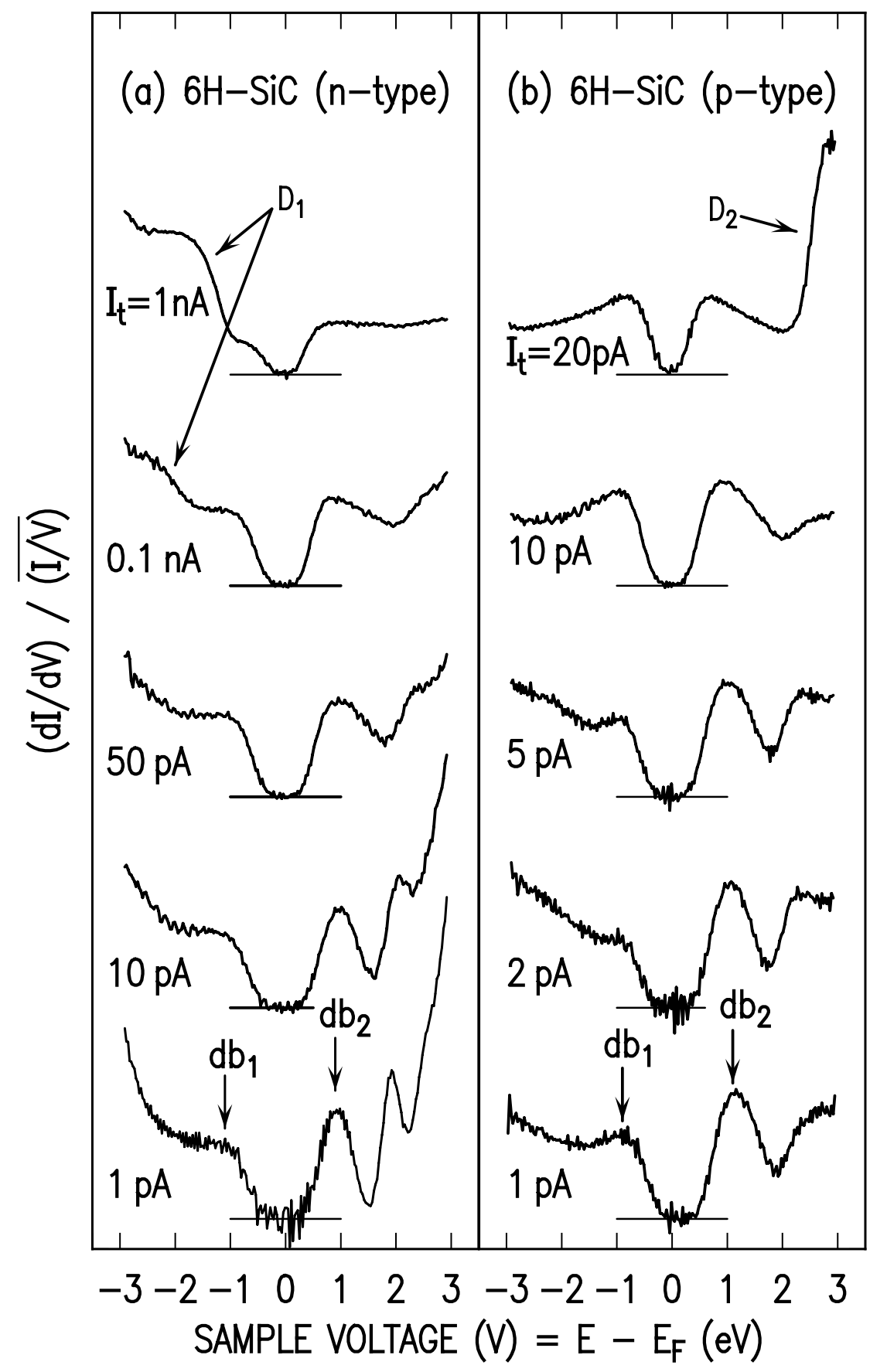

Figure 3 (a) A series of tunneling spectra with varying current setpoints (It t) taken on n-type 6HSiC. (b) A similar series taken on p-type material. The dopant induced current components, $D_{1}$ and $\mathrm{D}_{2}$, are labeled, as are the two states arising from the Si dangling bonds, $\mathrm{db}_{1}$ and $\mathrm{db}_{2}$. From Ref. [9]. 

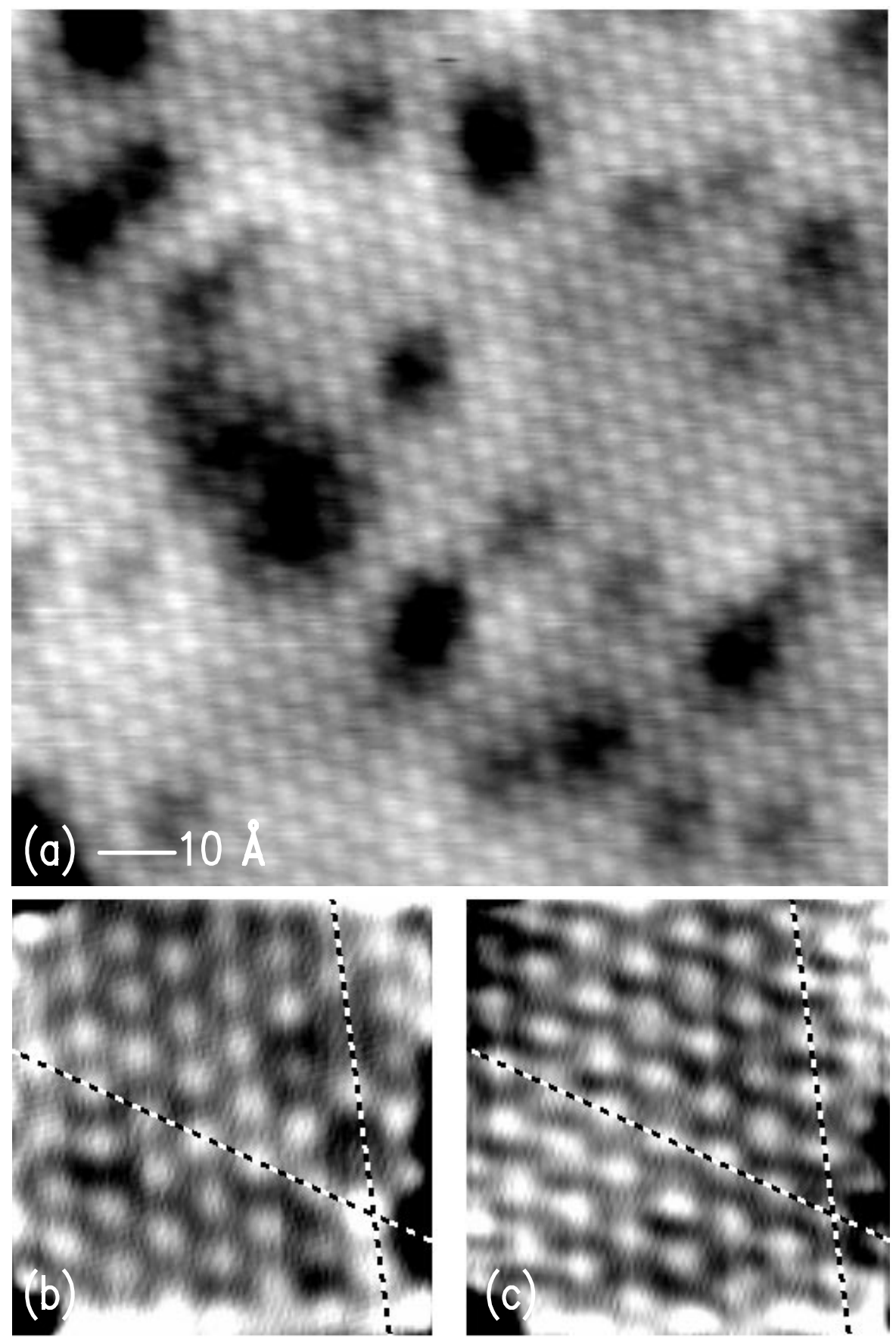

Figure 4 (a) Surface topograph of the $6 \mathrm{H}-\mathrm{SiC}(0001) \sqrt{3} \times \sqrt{3}$ surface taken at sample voltage of $1.2 \mathrm{~V}$ and $100 \mathrm{pA}$ current setpoint. The grey-scale ranges from 0 (black) to $0.6 \AA$ (white). (b) and (c) surface topographs $40 \AA \times 40 \AA$ in size taken at $-1 \mathrm{~V}$ and $+1 \mathrm{~V}$ respectively. Grey-scale range is $0.15 \AA$ and current setpoint was $10 \mathrm{pA}$. The two dashed intersecting lines in are drawn over two intersecting rows of topographic maxima, occurring at identical locations in the two image. From Ref. [9]. 

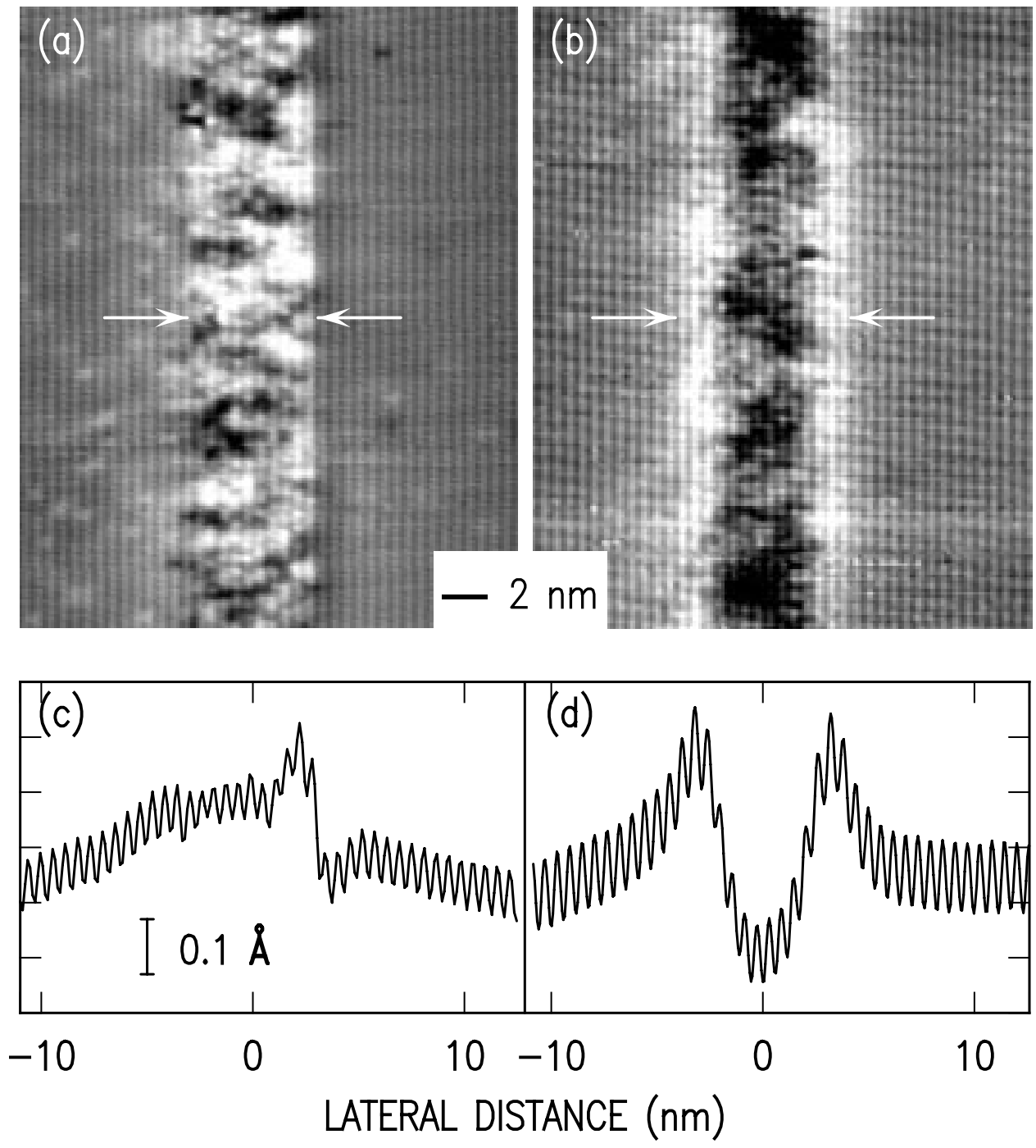

Figure 5 Cross-sectional STM images of InGaAs/InP heterostructures, (a) as-grown sample; (b) implanted and annealed sample. Images were acquired at sample voltages of +2.5 and $+2.0 \mathrm{~V}$ respectively, and grey scale ranges are 0.05 and $0.06 \mathrm{~nm}$ respectively. An average of the topographic line scans is shown in (c) and (d). Layer growth direction is from right to left. Arrows indicate approximate width of the quantum wells. From Ref. [39]. 


\section{(a) Electronic}

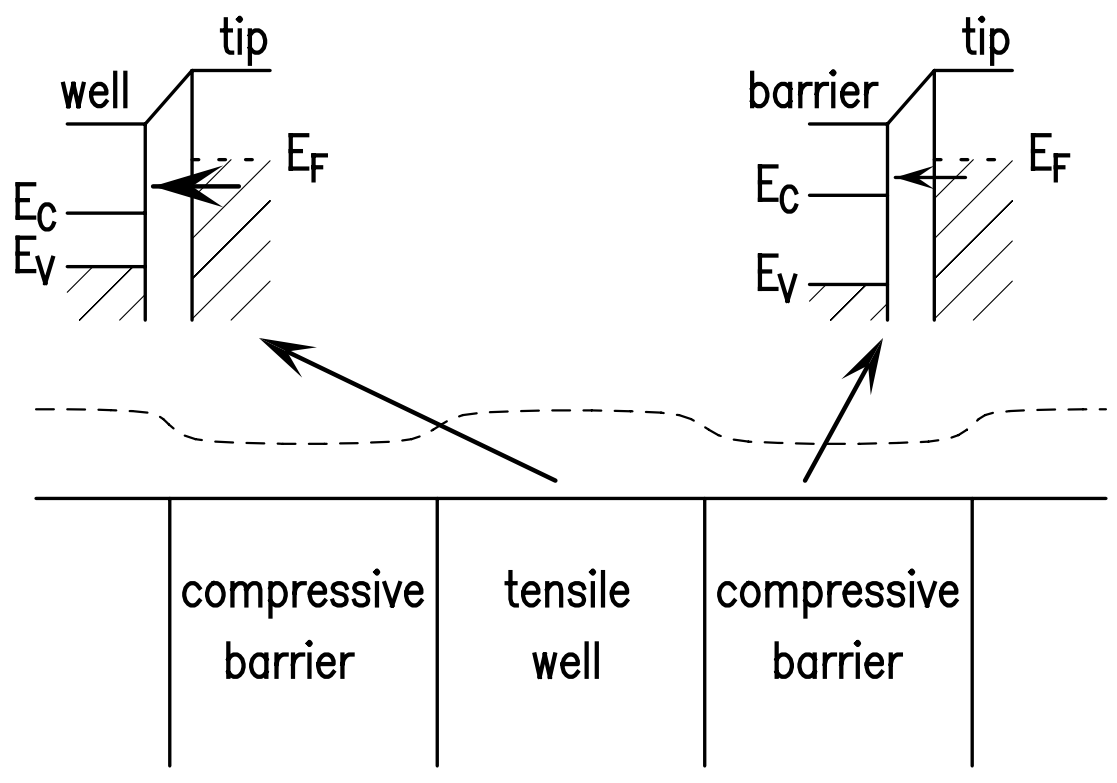

\section{(b) Mechanical}

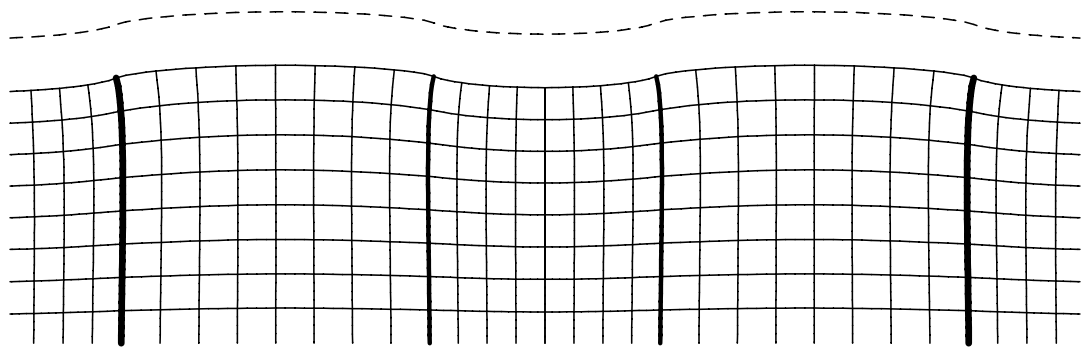

Figure 6 Illustration of STM contrast mechanisms for a strained semiconductor superlattice containing quantum wells in tension and barrier layers in compression. The dashed line shows the constant-current contour followed by the STM probe tip considering (a) only electronic, and (b) only mechanical effects. For case (a), the barrier has a larger band gap than the quantum well, so for a given tip-sample voltage there are fewer states available for tunneling to the barrier. Thus, a lower current is produced for a fixed tip-sample separation, so that the tip moves towards the sample to maintain a constant tunnel current. For case (b), relaxation of the strain produces an undulating surface morphology across the superlattice. For a strain of $\pm \varepsilon$ in the layers and width of both barrier and well of $L$, the peak-to-peak amplitude $2 h$ of the undulations is computed by finite elements to be $h / \varepsilon L \approx 1.0$ for Poisson ratio of 0.35. From Ref. [43]. 


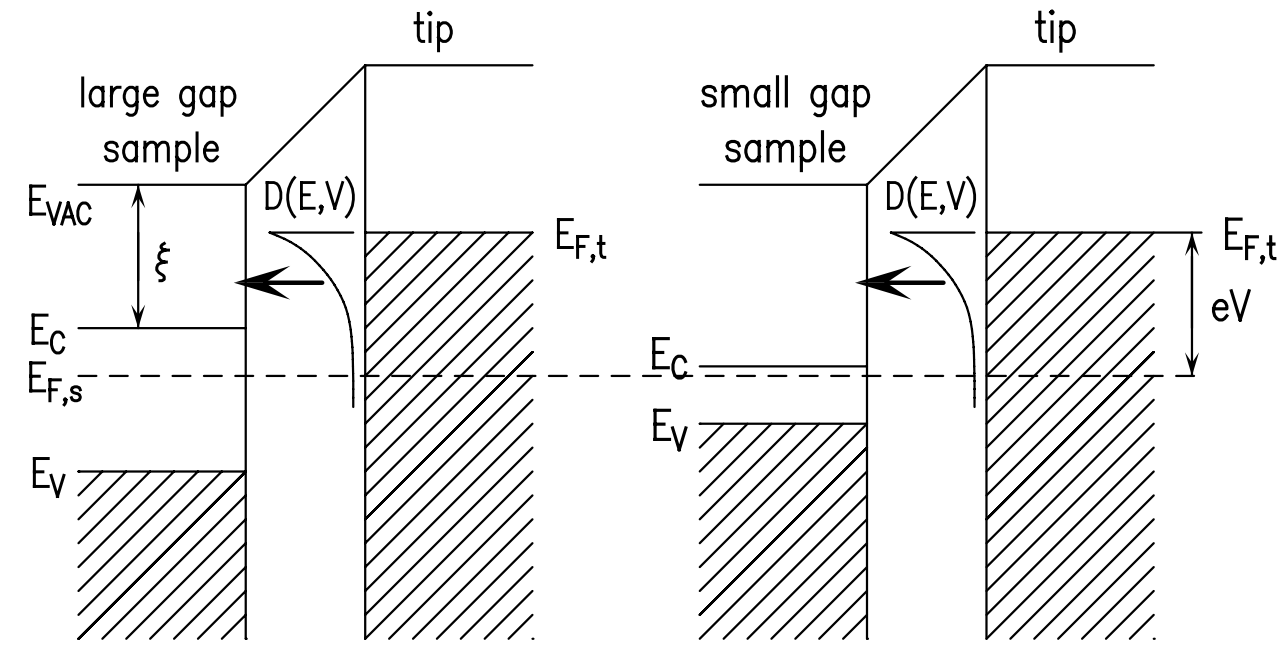

Figure 7 Energy level diagram for the case of tunneling into two neighboring semiconductor materials, with aligned Fermi levels, $E_{F, s}$ The probe tip Fermi level is denoted by $E_{F, t}$, and is separated from the sample Fermi level by $e V$ where $V$ is the applied voltage. The vacuum level of the sample is denoted by $E_{V A C}$ and electron affinity by $\xi$. The tunneling transmission term is indicated by $D(E, V)$. From Ref. [10].

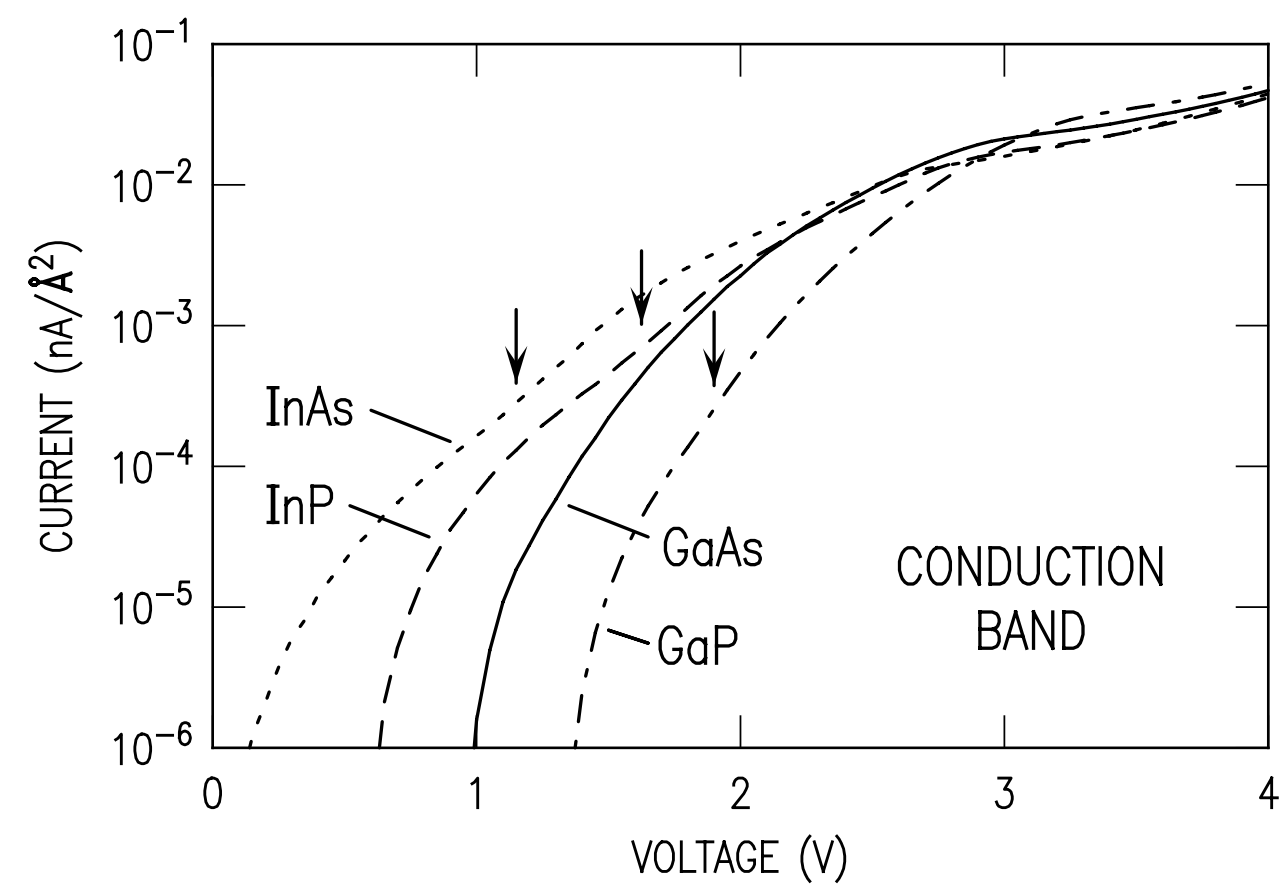

Figure 8 Computed tunnel current for CB states of GaAs, InP, InAs, and GaP. Arrows indicate the location of inflection points seen for InAs and InP, and seen weakly for GaP. From Ref. [10]. 